\title{
Ares I Flight Control System Overview
}

\author{
Charles Hall, Chong Lee, Mark Jackson, Mark Whorton, Mark West ${ }^{1}$ \\ NASA Marshall Space Flight Center, Huntsville, AL 35812 \\ Jay Brandon ${ }^{2}$ \\ NASA Langley Research Center, Hampton, VA 23681
}

Rob A. Hall, Jimmy Jang, Naz Bedrossian ${ }^{3}$

The Charles Stark Draper Laboratory, Inc.

\author{
Jimmy Compton ${ }^{4}$ \\ Dynamic Concepts, Inc. \\ Chad Rutherford ${ }^{5}$ \\ BD Systems, Inc.
}

\begin{abstract}
This paper describes the control challenges posed by the Ares I vehicle, the flight control system design and performance analyses used to test and verify the design. The major challenges in developing the control system are structural dynamics, dynamic effects from the powerful first stage booster, aerodynamics, first stage separation and large uncertainties in the dynamic models for all these. Classical control techniques were employed using innovative methods for structural mode filter design and an anti-drift feature to compensate for translational and rotational disturbances. This design was coded into an integrated vehicle flight simulation and tested by Monte Carlo methods. The product of this effort is a linear, robust controller design that is easy to implement, verify and test.
\end{abstract}

\section{Introduction}

The Ares I vehicle stack consists of a modified, Shuttle heritage first stage Solid Rocket Booster (SRB), a new upper stage to be developed in-house at the Marshall Space Flight Center, the service and crew modules (Orion) and a Launch Abort System solid motor extending from the top of the crew module. On the pad, the vehicle is comparable in height to the Saturn V launch vehicle, but much more slender. Characteristic of such a design are low structural mode frequencies that require special attention when designing bending filters for the control system. This is not an uncommon problem in launch vehicle control system design, but challenging nonetheless especially when the control designers job is not only to keep the vehicle stable in flight, but provide accurate guidance command tracking so the proper orbit insertion target can be reached.

While the first stage SRB has been employed for years on the Space Shuttle, its higher thrust derivative applied as the first stage for an in-line launch vehicle such as Ares I highlights interesting dynamic effects not considered very significant for Shuttle flight control. When the nozzle is gimbaled from the null position, the thrust may not be directed perfectly along the geometric center of the nozzle and the effective gimbal center may be offset from the centerline of the booster by a small amount causing roll, pitch and yaw torques that must be compensated for by the control system. These effects were known to exist for the Shuttle SRB but their influence is minimal since the Shuttle stack employs two boosters in parallel and has three liquid engines as well to counter such disturbance torques.

\footnotetext{
${ }^{1}$ Aerospace Engineers, Flight Mechanics and Analysis Division/EV40, Huntsville, AL 35812.

${ }^{2}$ Aerospace Engineer, /RTD-Flight Dynamics, Hampton, VA . 23681

${ }^{3}$ Aerospace Engineers, /,

${ }^{4}$ Aerospace Engineer, /,

${ }^{5}$ Aerospace Engineer, /,
} 
The first stage Roll Control System (RoCS) had to be designed to deal with thrust vector and aerodynamic disturbance torques while providing steering torque for roll maneuvers commanded by guidance. The upper stage reaction control system (ReCS) has to deal with large attitude errors and rates after first stage separation. After the upper stage engine cuts off, three axis control is provided by the ReCS to damp roll pitch and yaw body rates until Orion separates. These effects had to be considered in the design of the phase planes to provide adequate roll attitude orientation and rate damping while conserving propellant.

The design philosophy for the Ares I flight control system (FCS) was to draw from previous flight experience to establish a control architecture to begin with, then modify as needed to address vehicle and mission specific requirements and design goals. Studying Saturn/Apollo vehicles and control systems revealed that the first bending mode frequency was not far from that of Ares I, which led to adopting a proportional-integral-derivative control law with serial bending filters to phase stabilize the first bending mode. Consultation with private industry launch providers led to investigating the use of distributed rate gyros to increase robustness of the control system to vehicle bending during high dynamic pressure regions of flight. Load relief systems from Saturn and Shuttle were analyzed and tested in simulations of the Ares I, as well as a new anti drift channel to increase robustness to force and torque disturbances such as those caused by wind and thrust vector misalignment. Shuttle experience led to the development of an efficient phase plane controller for the RoCs and ReCS. Rigorous stability analyses and flight simulations have shown that the current FCS design has proven to be robust to large system dispersions and vehicle design uncertainties.

\section{Vehicle Description}

Figure 1 shows and expanded view of Ares I. The first stage is a single, recoverable solid rocket booster developing roughly 3.5 million pounds of peak thrust at about 83 thousand feet for a nominal mission. It will use Shuttle SRB heritage TVC actuators. A redundant Rate Gyro Unit (RGA) housed in the aft skirt is used for flight control. Roll control for first stage is managed by the RoCS mounted in two external pods on the interstage, which also houses a second RGA package used for flight control. The upper stage is powered by a single liquid propellant engine, the J-2X, that develops 294,000 lbf vacuum thrust. The upper stage ReCS, providing roll, pitch and yaw control, is located in two external pods on the aft end of the upper stage. The Inertial Measurement Units (IMU) and flight computers used for guidance, navigation and control are located in the instrument unit at the top of the upper stage.

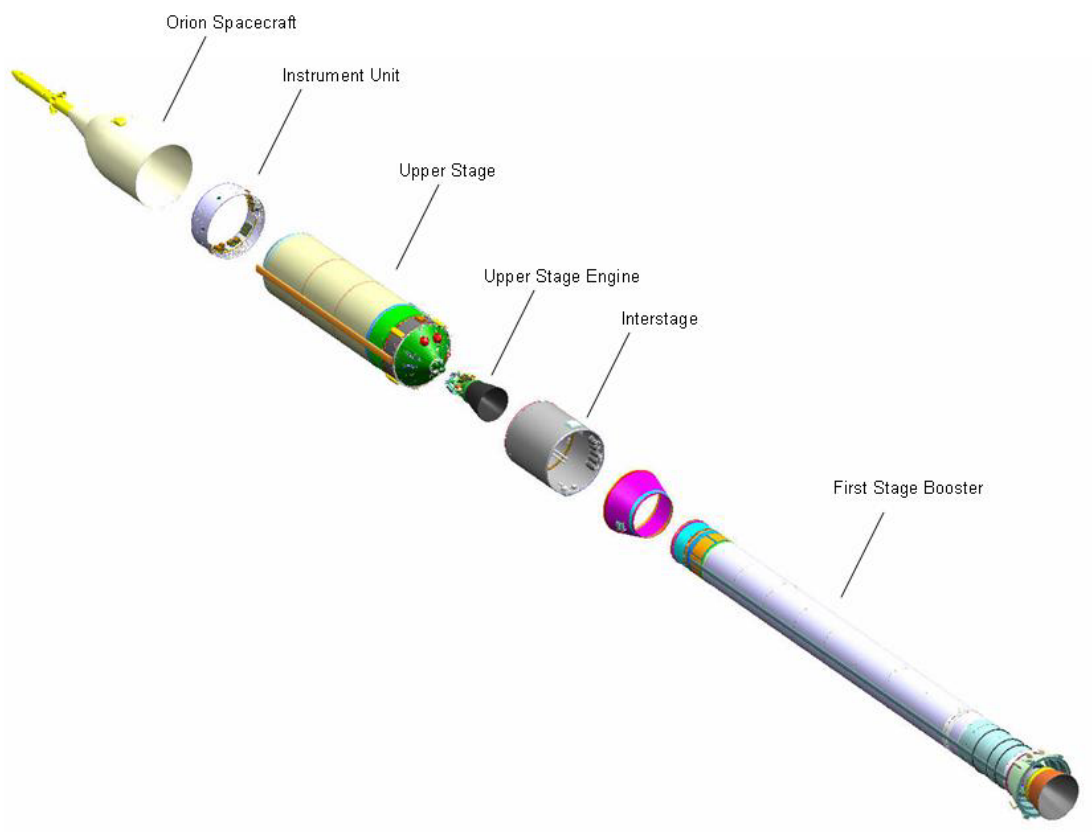

Figure 1: The Ares I Launch Vehicle. 


\section{Flight Control System Description}

The FCS uses a Thrust Vector Control (TVC) system consisting of two actuators located 90 degrees apart that position the first and second stage nozzles for pitch and yaw control. Roll control for powered flight is provided by two separate systems of thrusters located in two pods, 180 degrees apart, on the interstage and on the upper stage. The upper stage thrusters also provide roll, pitch and yaw rate damping after the upper stage engine cuts off. The IMU provides an attitude quaternion and body rates to the FCS. Pitch and yaw body rates are also obtained from the RGU packages located forward in the interstage and aft in the SRB skirt. The control algorithms for first and second stage flight are identical, except for the three axis capability of the ReCS for post-MECO coast.

A block diagram of the pitch/yaw control system is shown in Figure 2. Pitch and yaw control channels are identical thanks to vehicle symmetry, with one producing a gimbal command in the pitch body axis and the other in the yaw body axis. These signals are then sent to a mixing and limiting logic (not shown) that provides command to the TVC actuators. Guidance provides steering commands in the form of a command quaternion which the FCS combines with the vehicle attitude quaternion to obtain roll, pitch and yaw attitude errors. Guidance also provides angular rate commands that are summed with sensed body rates to form rate errors. Sensed body rates and axial acceleration are provided by the IMU. The forward and aft RGA packages provide pitch and yaw body rates only.

The error signals are filtered in two stages, first with a notch filter then with a low pass filter. These filters provide the necessary phase and gain shaping required to stabilize the bending modes. After filtering the rate errors, the signals are gained and summed in such a way as to blend the forward and aft signals to reduce bending mode amplification of the rate signals due to structural bending. More discussion on filter design and sensor placement will be discussed in $\S$ IV, Stability Analysis. The filtered and gained error signals are sent to a proportional-integralderivative (PID) control law designed to provide the proper bandwidth as well as good transient and steady state responses. This signal is filtered again with a low pass filter after inputs from the anti-drift channel are added. Finally, a loop gain is applied and the signal is negated for proper polarity to obtain the final gimbal command.

The anti-drift (AD) algorithm includes force and moment balances using input data from rate gyros and accelerometers. The AD design maintains the same response to control as the normal PID control system, and has little effect on the PID control gain and phase margins. Signals from the AD algorithm augment those from the PID algorithm to help reduce translational drift from the trajectory due to wind, TVC biases, and center of gravity (CG) offsets. Two outputs come from the AD block - an angle bias term, and a PID bias term. The angle bias term is used to align the thrust vector in a direction to balance external forces, and the PID bias term is an input used to balance external moments. The external forces and moments could be caused by aerodynamics, TVC offsets, gusts, winds, etc. The actual implementation includes some low-pass filtering to keep noise from causing undesired results, and to keep the response frequencies of the $\mathrm{AD}$ low to avoid coupling with the structural vibrations. Additionally, since the accelerometer data is from the IMU - which may have a radial offset from the centerline of the vehicle and not at the axial CG location, corrections to the acceleration signals are made due to body angular rates. The AD and PID control laws require scheduled gains that are related to thrust, inertia, CG location, and weight. 


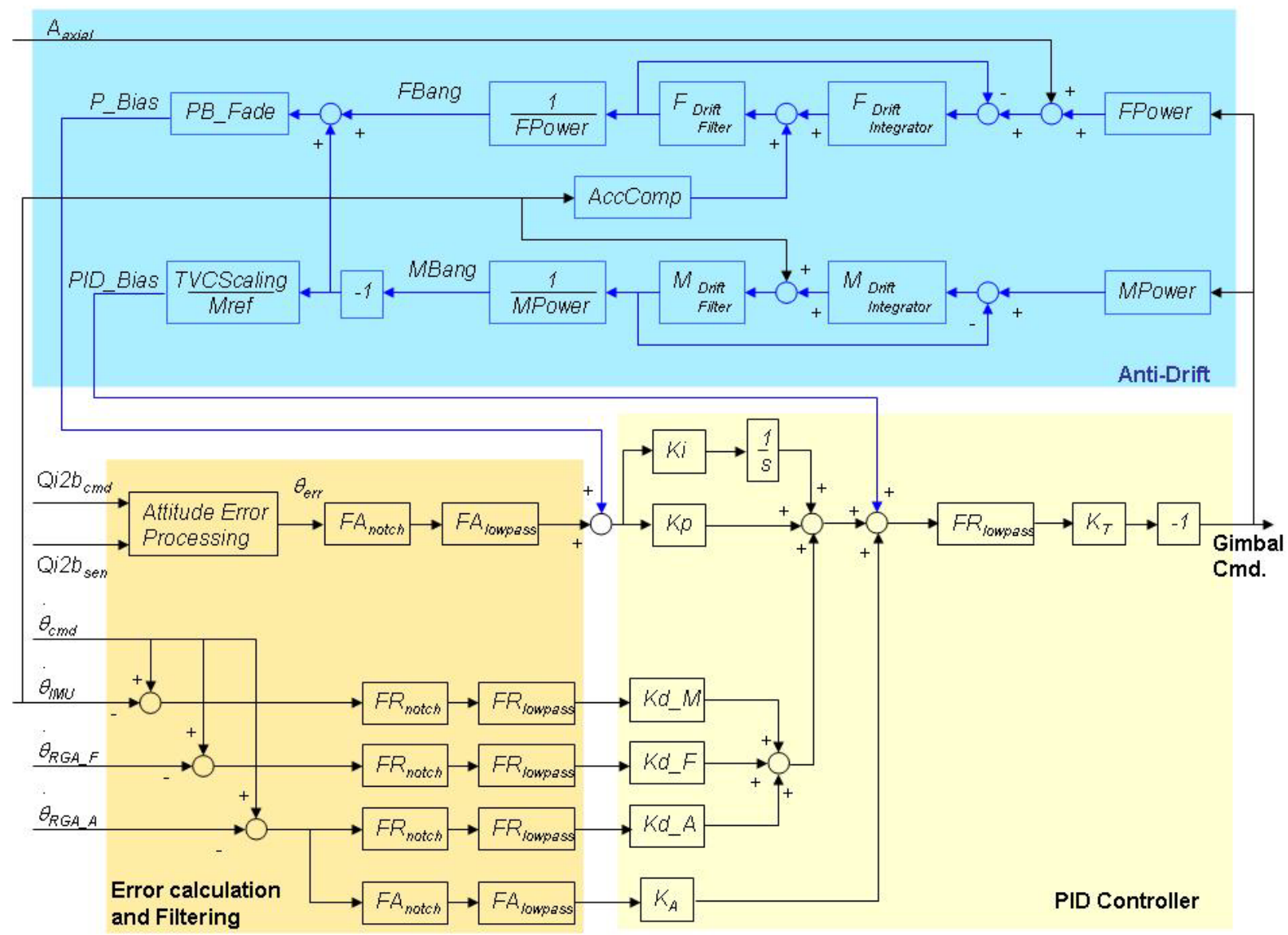

Figure 2: Pitch/Yaw system

The FCS uses phase plane autopilots for roll during powered phases and for roll, pitch and yaw during the post MECO coast phase of flight. The roll, pitch and yaw designs are identical, but use different data loads depending on the axis being controlled and the flight phase. Inputs to the phase plane are filtered attitude and body rate errors. Each phase plane autopilot produces a single command that takes on three states; 0 for drift, +1 for positive rotation and -1 for negative rotation. This command is sent to a jet selection logic that sets the appropriate RCS jet command(s) to "on" or "off". The phase plane design utilizes linear switching lines and hysteresis curves. A typical phase plane for the roll axis is illustrated in Figure 3. Pitch and yaw phase planes are similar. The drift channel is designed to minimize rate and hence may lead to propellant savings. The hysteresis curves and deadbands are added to reduce the number of firings and severity of potential limit cycle behavior in the presence of external disturbances. 


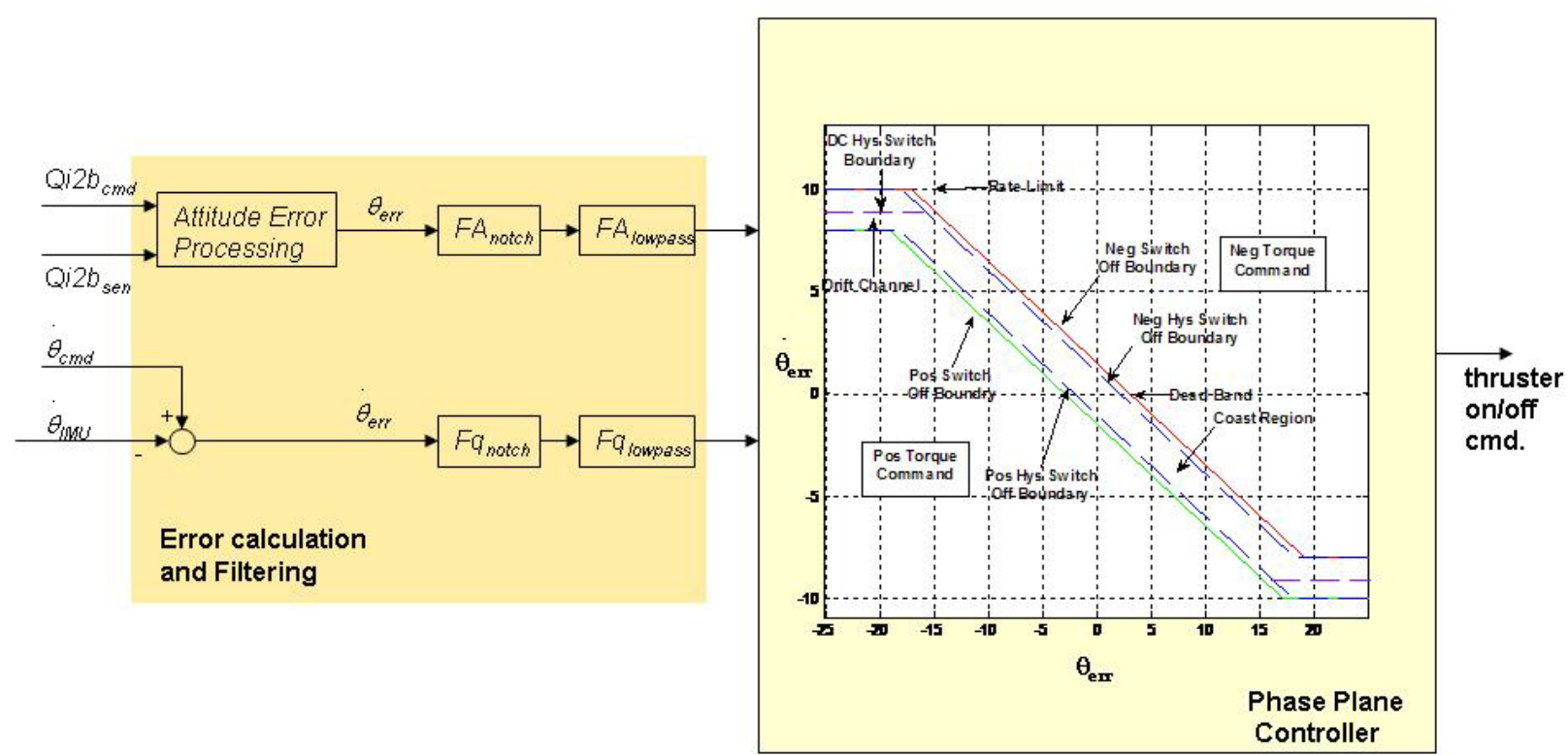

Figure 3: Phase plane controller.

\section{Stability Analysis}

Stability analyses were conducted in two stages, the first for the purpose of filter design and an initial assessment of control system robustness, and the second in a Monte Carlo analysis where all system dispersions are applied. Both analyses must meet stability margin requirements in gain and phase before the design is accepted for rigorous flight performance analysis in the time domain.

The filter design methodology was based on a numerical constrained optimization approach to maximize performance. The design parameters are the coefficients of bending filters. A set of feasible parameters must satisfy the following constraints:

1. The filter itself must be stable and minimum phase to guarantee stability and performance.

2. The bandwidth of the bending filter should be greater than that of the PID controller to avoid rigid performance degradation.

These constraints can be used to set the upper and lower bounds for the design parameters ${ }^{3}$.

Multiple rate sensors are used along the structure to allow for the blending of the sensed rotational rate, and hence active removal of flex dynamics in the rate channel ${ }^{4}$. Design of the rate gyro blending control law has two key control considerations:

1. The phase of the first bending mode must be well understood since the first mode is phase stabilized.

2. The gyro blending will aid in gain stabilization of the higher bending mode dynamics by flex rate cancellation.

For first mode phase stabilization, the sensor weighting is designed such that the upper stage sensors dominate the control law input, hence the first bending mode must be significantly lagged by the filtering to compensate for the sensors being non-collocated with the actuator. Specifically, the upper stage rate gyro is heavily weighted when blending with the first stage rate gyro. These blending weights are designed to significantly remove second mode flex intrusion, while robustly preserving the phase stabilization on the first flex mode. 
An example of first stage stability analysis results are shown in the Nichols plot shown in Figure 4. Second stage is less challenging due to higher bending modes and the absence of aerodynamics, so only first stage results will be shown. In this analysis, robustness of the pitch channel filter designed is examined. The first bending mode frequency was dispersed $\pm 10 \%$ and all higher modal frequencies were dispersed $\pm 30 \%$. Stability margin requirements are depicted as diamonds at the Nyquist points, where the black diamonds represent the dispersed requirements of $\pm 6 \mathrm{~dB}$ gain and 20 degrees phase and the magenta diamonds represent the nominal requirements of $\pm 6 \mathrm{~dB}$ gain and 30 degrees phase. Frequency response curves at 10 second intervals are shown, with lowest frequency on each curve at the upper right part of the plot and highest frequencies at the lower left. It can be seen that low frequency margins, where aerodynamics and propellant slosh are important, are being met. Although the first bending mode can have significant gain, it is phase stable while all the higher frequency modes are gain stable with ample margin.

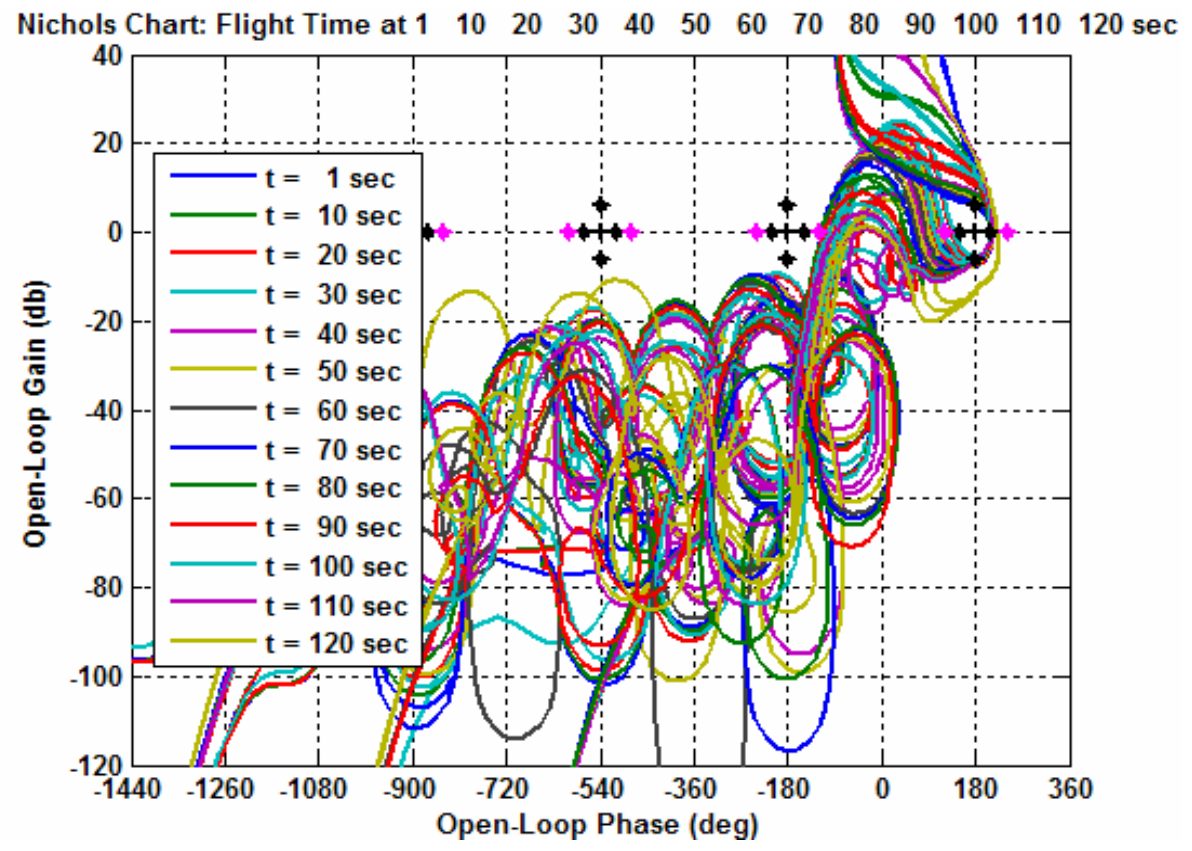

Figure 4: First stage Nichols plot with modal dispersions.

In the next phase of stability analysis, all system dispersions are applied that include not only bending mode dispersions, but dispersed aerodynamics, propulsion system, environment (wind), mass properties and sensors. An example of first stage margins are shown in Table 1.

Table 1. Dispersed stability margins for first stage flight.

\begin{tabular}{|c|c|c|c|c|c|c|}
\hline & \multicolumn{6}{|c|}{ ISS Trajectory } \\
\hline & Margin & $1 \mathrm{Sec}$ & $22 \mathrm{Sec}$ & $30 \mathrm{Sec}$ & $42 \mathrm{Sec}$ & $60 \mathrm{Sec}$ \\
\hline Pitch & Low Frequency GM & 13.9 & 12.6 & 10.7 & 8.7 & 6.4 \\
\hline Pitch & High Frequency GM & 9.7 & 9.8 & 10.2 & 10.2 & 8.8 \\
\hline Pitch & First Flex Mode GM & 20.0 & 55.3 & 54.6 & 58.6 & 20.0 \\
\hline Pitch & Low Frequency PM & 32.0 & 40.0 & 40.3 & 40.3 & 33.7 \\
\hline Pitch & First Flex Mode PM & 55.8 & 59.0 & 60.9 & 61.8 & 57.2 \\
\hline Yaw & Low Frequency GM & 13.9 & 12.6 & 10.7 & 8.8 & 6.3 \\
\hline Yaw & High Frequency GM & 9.7 & 9.8 & 10.3 & 10.2 & 8.8 \\
\hline Yaw & First Flex Mode GM & 20.1 & 55.3 & 54.8 & 56.9 & 20.4 \\
\hline Yaw & Low Frequency PM & 36.2 & $\begin{array}{l}40.0 \\
6\end{array}$ & 40.2 & 40.0 & 33.7 \\
\hline
\end{tabular}

American Institute of Aeronautics and Astronautics 


\begin{tabular}{|c|c|c|c|c|c|c|}
\hline Yaw & First Flex Mode PM & 55.7 & 58.9 & 60.8 & 61.6 & 57.1 \\
\hline & Margin & $85 \mathrm{Sec}$ & $105 \mathrm{Sec}$ & $110 \mathrm{Sec}$ & $118 \mathrm{Sec}$ & $120 \mathrm{Sec}$ \\
\hline Pitch & Low Frequency GM & 9.4 & 13.9 & 18.2 & 16.8 & 13.8 \\
\hline Pitch & High Frequency GM & 9.1 & 8.8 & 8.0 & 14.0 & 17.3 \\
\hline Pitch & First Flex Mode GM & 24.0 & 26.4 & 28.4 & 36.8 & 40.2 \\
\hline Pitch & Low Frequency PM & 34.7 & 33.6 & 36.2 & 43.8 & 42.6 \\
\hline Pitch & First Flex Mode PM & 64.5 & 71.1 & 75.2 & 109.9 & Inf \\
\hline Yaw & Low Frequency GM & 9.4 & 13.9 & 18.1 & 16.8 & 13.8 \\
\hline Yaw & High Frequency GM & 9.1 & 8.7 & 8.2 & 13.9 & 17.4 \\
\hline Yaw & First Flex Mode GM & 23.7 & 26.1 & 28.1 & 36.9 & 40.3 \\
\hline Yaw & Low Frequency PM & 34.6 & 32.0 & 34.5 & 43.7 & 42.5 \\
\hline Yaw & First Flex Mode PM & 64.3 & 71.1 & 74.2 & 109.9 & Inf \\
\hline
\end{tabular}

\section{Simulated Flight Performance Analysis}

The FCS algorithms are straightforward and easy to implement is a computer simulation. The flight simulation code used for the Ares I project, MAVERIC (Marshal Aerospace VEhicle Representation In C) [], contains detailed vehicle, subsystem and environmental models and is used for single runs as well as large batches of runs for Monte Carlo analysis. At this stage of Ares I development, the vehicle design is evolving therefore math models representing the vehicle have fairly large uncertainties associated with them. A description of the dispersions may be found in the reference ${ }^{5}$. Applying these large uncertainties along with known wind dispersions during the most severe months is a good test for control system robustness.

Ares I has two reference trajectories, one to carry crew and payload to an insertion target for the International Space Station (ISS) and the other is a space exploration trajectory referred to as the Due East (DE) trajectory. Since mass estimates among the vehicle elements are still converging, to adequately cover all scenarios two different vehicles have to be simulated; a heavy/slow vehicle and a light/fast vehicle. Certain months are known to be demanding not only on the control system but on loads, heating rates and upper stage re-entry footprint. This culminated in nine different Monte Carlo cases, each having 2,000 individual simulations in order to capture three sigma dispersions with the required confidence level. To further test control system robustness, the gains and filters were designed for only one mean reference trajectory and the nominal vehicle. Only one filter coefficient set for first stage and one set for second stage were used.

The dispersed simulations are analyzed to investigate many things, such as aerodynamic and thermal loads, mass to orbit, insertion accuracy and control system performance to name a few. Some example control system parameters are shown in figures 5 through 9 as envelope plots where the minimum and maximum values at each time point from all 2,000 simulations are shown as two continuous curves (blue), co-plotted with the same parameter from a nominal simulation (red curve). From these nine sets of Monte Carlo simulations, the most demanding ones for the control system and its hardware were singled out. The largest roll attitude error (figure 5) occurs during the ISS mission in August due to the large roll maneuver required concurrent with roll disturbances arising from thrust vector coupling and in this case, a hot, higher thrust SRB. The roll control system performs the roll maneuver and brings the attitude back within the two degree dead band for the remainder of the flight. The pitch and yaw attitude errors show how wind disturbances during high dynamic pressure regions of flight can cause the vehicle to diverge from it's trajectory, although peak errors are very reasonable. First stage guidance is open loop and obtains steering commands from a table []. The guidance commands are derived from a gravity turn trajectory that steers the vehicle so that aerodynamic angles of attack and side slip are zero before maximum aerodynamic pressure (max Q) is reached. The control system follows this steering even in the face of wind and other disturbances and reliably brings aerodynamic angles to small values just before max $\mathrm{Q}$ which occurs around 60 seconds. The only demanding part of second stage flight is right after first stage separation when the control system must remove attitude errors that build during the SRB separation event. Guidance is closed loop during upper stage flight, and immediately begins to command the attitude and rates to steer to the required insertion target. TVC actuator gimbal angle envelope plots for first and upper stage flight are shown in Figures 8 and 9, respectively. The TVC actuators are skewed 90 degrees 
from the vehicle body pitch and yaw axes and are named rock and tilt for this reason. The actuator maximums and minimums correlate directly with the attitude errors as the control system commands them to remove the errors. The deflections are well within design capability. Figure 10 typifies another format used in analysis of Monte Carlo simulations. They show scatter plots of RCS propellant used at first stage separation on the left, and upper stage RCS propellant used at MECO on the right. The ordinate is propellant and the abscissa is the corresponding run number. From this type of plot, a visual feel can be had for the mean value and the outliers can be easily seen. By zooming in on the plot, the run number corresponding to a maximum or minimum can be found and that simulation examined in detail. These plots revealed that RCS propellants used were well within design limits.
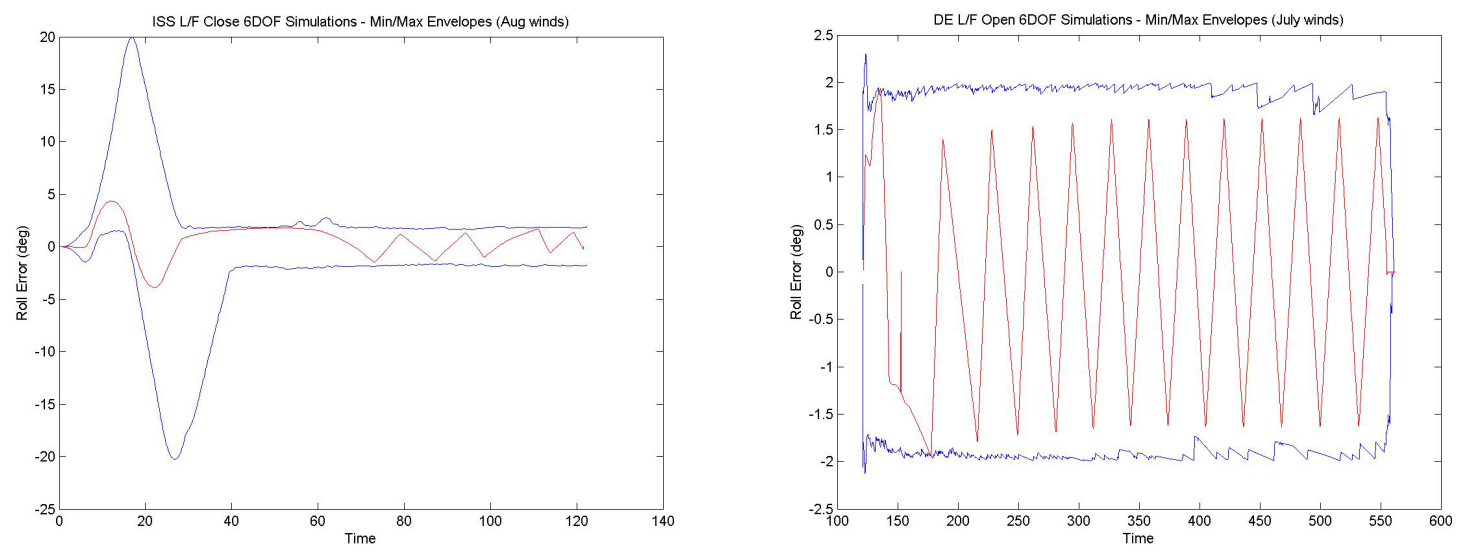

Figure 5: First and upper stage roll attitude error.
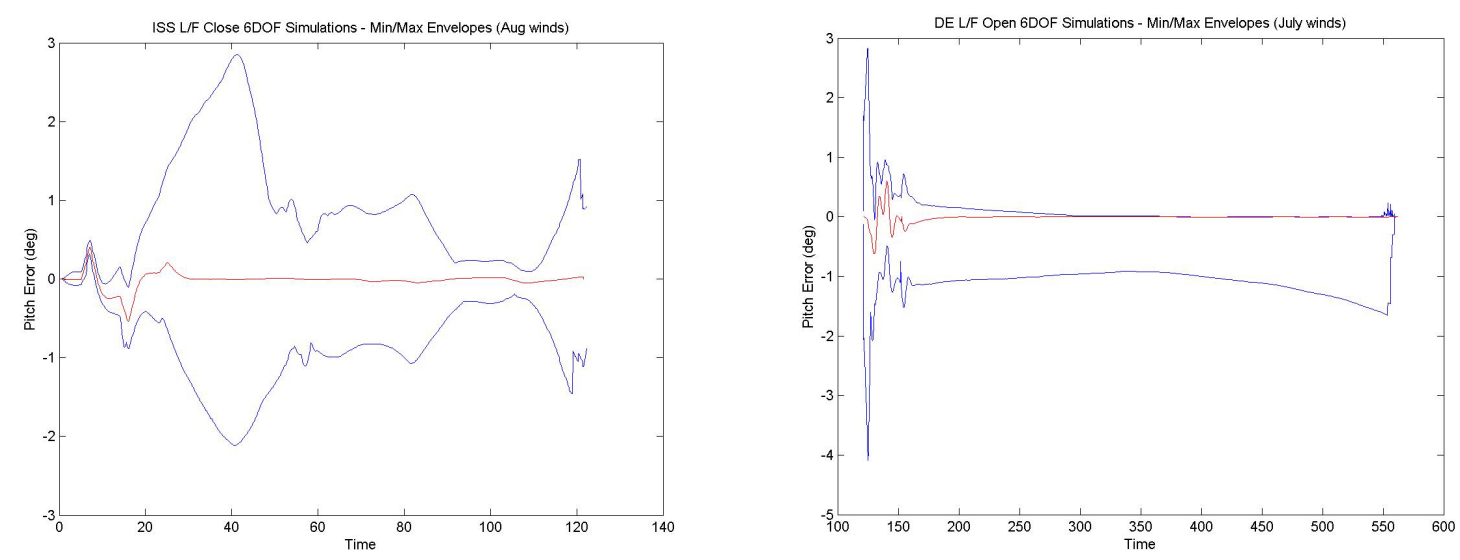

Figure 6: First and upper stage pitch attitude error. 

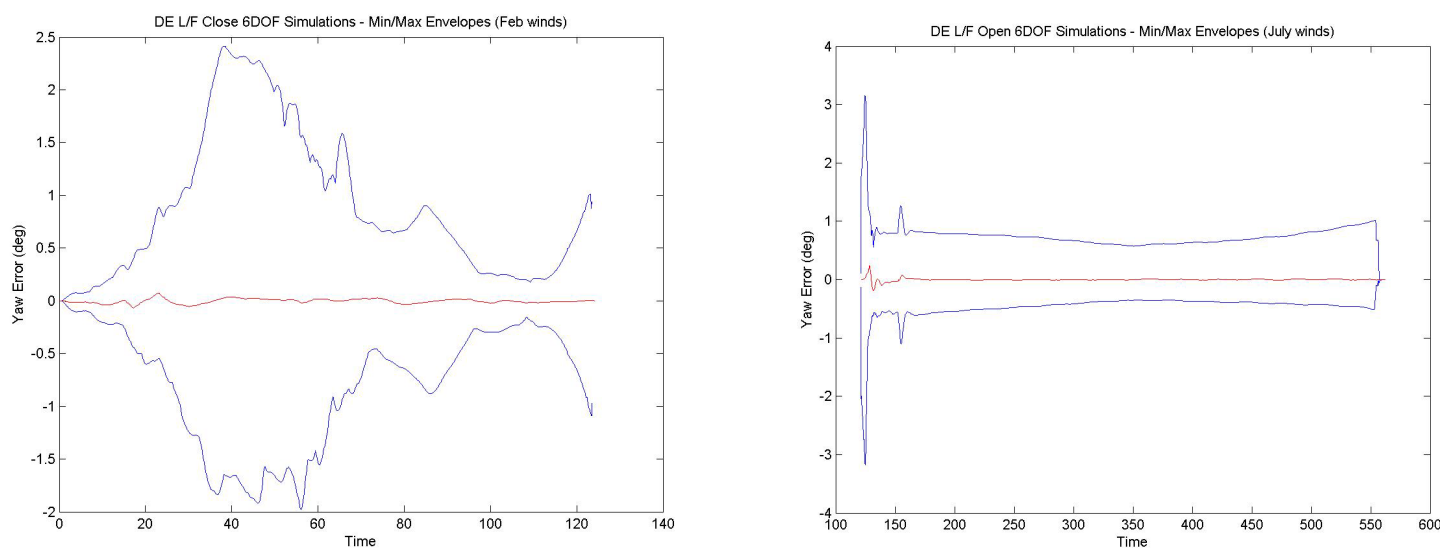

Figure 7: First and upper stage yaw attitude error.
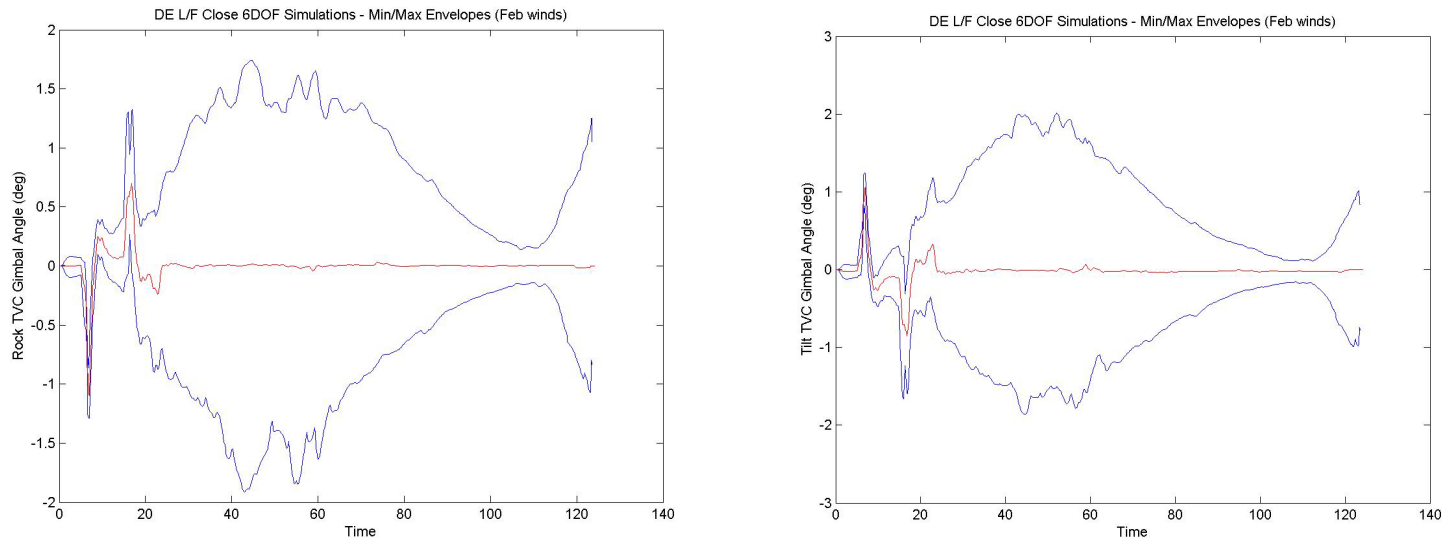

Figure 8: First stage rock and tilt gimbal angles.
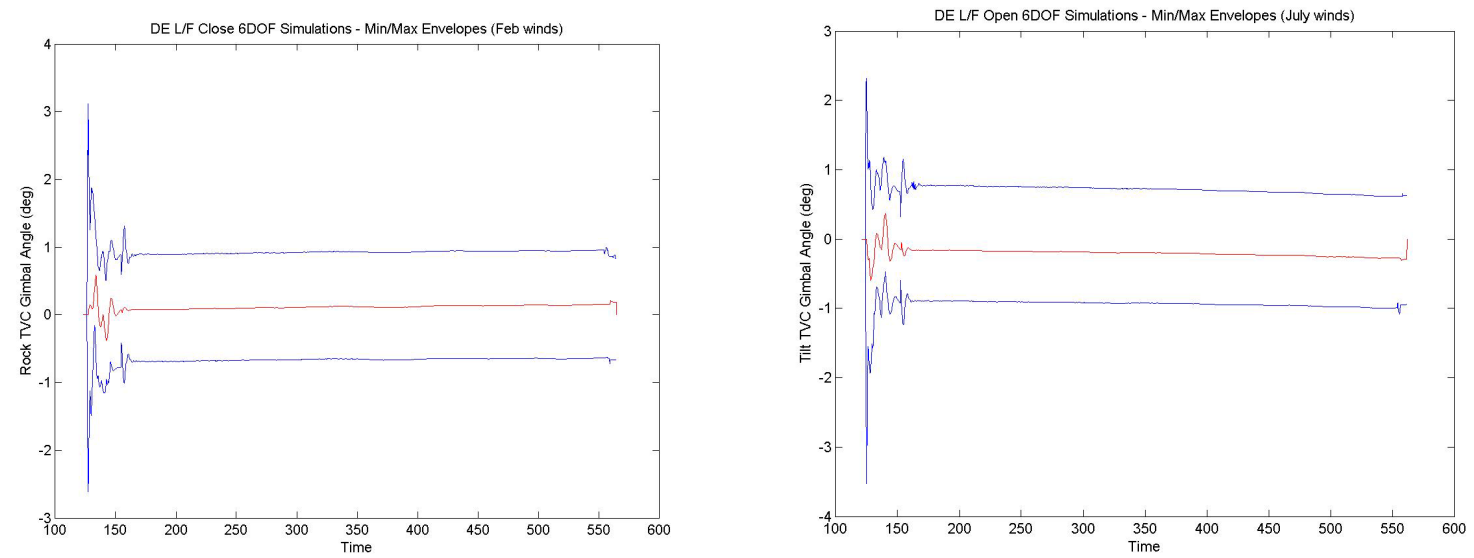

Figure 9: Upper stage rock and tilt gimbal angles. 

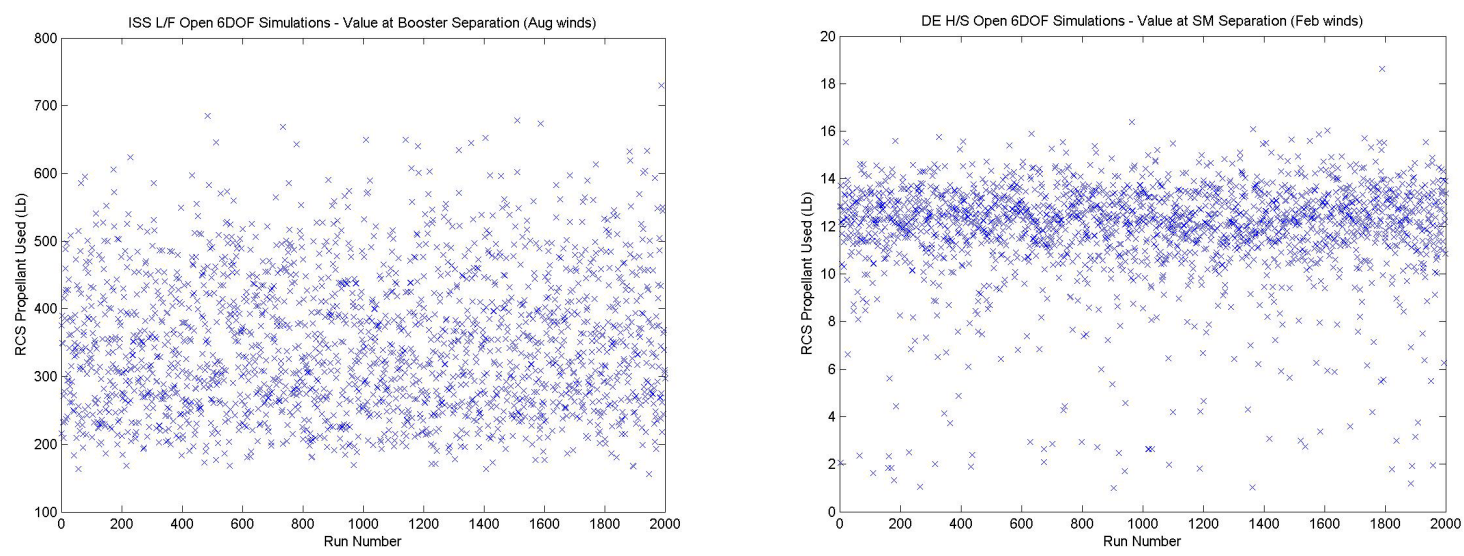

Figure 10: First and upper stage RCS propellant scatter plots.

\section{Conclusion}

Classical control techniques, complimented by innovative bending filter design techniques and an anti-drift feature have produced a robust linear control system for the Ares I launch vehicle that is easy to implement, verify and test in flight simulations. Extensive stability analyses verify stability in the frequency domain while non-linear, high fidelity flight simulations verify good performance in the time domain. The design proved to be robust to very large dispersions in vehicle and environmental models, while using gain and filter sets designed for a single, mean trajectory and nominal vehicle.

\section{References}

${ }^{1}$ Ares I Guidance papers

${ }^{2}$ Ares I Flight Control papers

${ }^{3}$ Jang, J., Bedrossian, N., Lee, A, Spanos, P., "A Constrained Optimization Approach for CMG Robust Flex Filter Design", AIAA GN\&C Conference, August 2002.

${ }^{4}$ Garner, D., "Control Theory Handbook", NASA Marshall Space Flight Center, NASA TM X-53036, April 22, 1964.

${ }^{4}$ Maveric reference

${ }^{5}$ Ares I Monte Carlo paper reference 